\title{
Right Iliac Artery-Inferior Vena Cava Arteriovenous Fistula
}

\author{
Umberto G. Rossi, MD ${ }^{1,2}$ Pierluca Torcia, MD² Gian Andrea Rollandi, MD ${ }^{3}$ Maurizio Cariati, MD 2 \\ ${ }^{1}$ Department of Diagnostic Imaging - Interventional Radiology Unit, \\ Galliera Hospital, Genova, Italy \\ 2 Department of Diagnostic Science - Radiology and Interventional \\ Radiology Unit, ASST Santi Paolo and Carlo Hospital, Milano, Italy \\ Address for correspondence Umberto G. Rossi, MD, EBIR, \\ Department of Diagnostic Science - Interventional Radiology Unit, \\ Galliera Hospital, Mura delle Cappuccine, 14 - 16128, Genova, Italy \\ (e-mail: urossi76@hotmail.com; umberto.rossi@galliera.it).
}

${ }^{3}$ Department of Diagnostic Imaging - Radiology Unit, Galliera

Hospital, Genova, Italy

AORTA 2018;6:46-47.
Abstract Keywords
- right iliac artery
- inferior vena cava
- arteriovenous fistula
- pseudoaneurysm
- elderly patient (aging)

This report describes a case of elderly patient with right iliac artery fissured pseudoaneurysm, leading to inferior vena cava arteriovenous fistula that was treated by covered stent device deployed at the level of the right iliac pathologic segment.
A 77-year-old male patient underwent open repair aorto-biiliac graft for abdominal aortic aneurysm (AAA) 7 years ago. On clinical follow-up, an abdominal bruit was observed. The patient underwent contrast medium-enhanced multidetector computed tomography (MD-CT) that revealed in the arterial phase, at the level of the right iliac anastomosis, the presence of fissured pseudoaneurysm (arrowhead in - Fig. 1) in communication with the proximal third of the inferior vena cava, with a rapid contrast filling of an enlarged inferior vena cava (shown by * in - Fig. 1). So, a diagnosis of right iliac artery-inferior vena cava arteriovenous fistula (AVF) was made. After multidisciplinary agreement, the patient underwent digital subtraction angiography that confirmed the diagnosis of fissured pseudoaneurysm (arrowhead in -Fig. 2), leading to right iliac artery-inferior vena cava AVF with inferior vena cava enlargement (shown by * in - Fig. 2). Consequently, a covered stent device was deployed as arterial coverage at the level of the fissured pseudoaneurysm of the right iliac anastomosis with the resolution of the AVF. The patient at 1-year follow-up demonstrated clinical and technical successes.

Iliac artery-inferior vena cava or iliac vein AVF, due to iliac anastomotic pseudoaneurysm, is a rare clinical complication after AAA open repair. ${ }^{1}$ Contrast medium-enhanced MD-CT is the first-line imaging modality to confirm the diagnosis and to plan the possible repair procedure in a suspected iliac arteryinferior vena cava or iliac vein $\mathrm{AVF}^{2}$ AVF as complication of

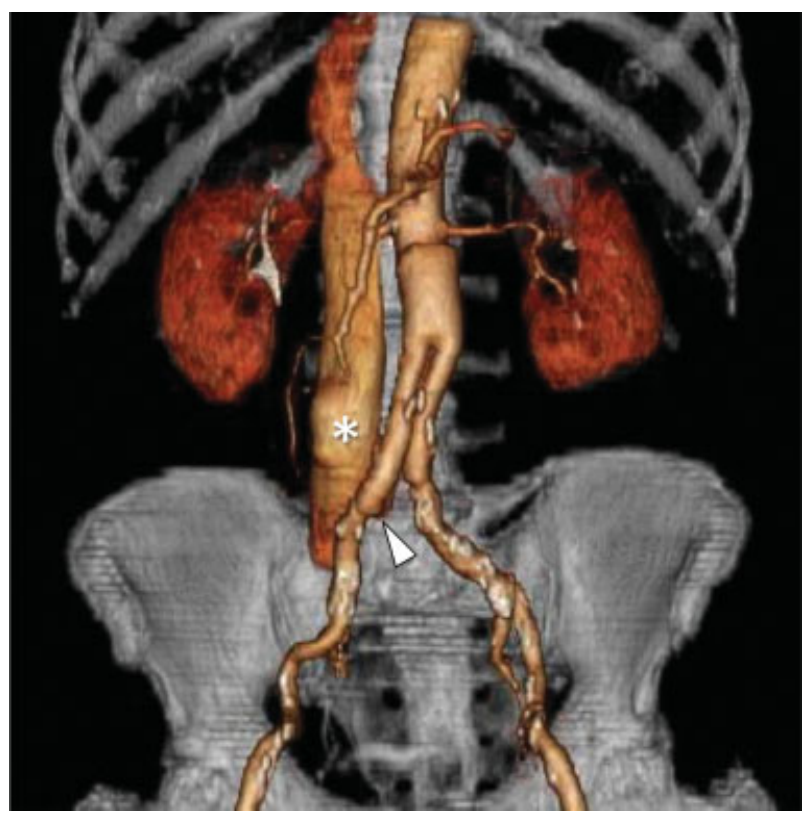

Fig. 1 Abdominal contrast medium-enhanced multidetector computed tomography coronal volume rendering technique reconstruction that shows, at the level of the right iliac anastomosis, the presence of fissured pseudoaneurysm (arrowhead) in communication with the proximal third of the inferior vena cava $\left({ }^{*}\right)$.
DOI https://doi.org/ 10.1055/s-0038-1639611. ISSN 2325-4637.
Copyright $\odot 2018$ by Thieme Medical Publishers, Inc., 333 Seventh Avenue, New York, NY 10001, USA. Tel: +1(212) 584-4662.
License terms

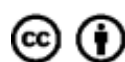




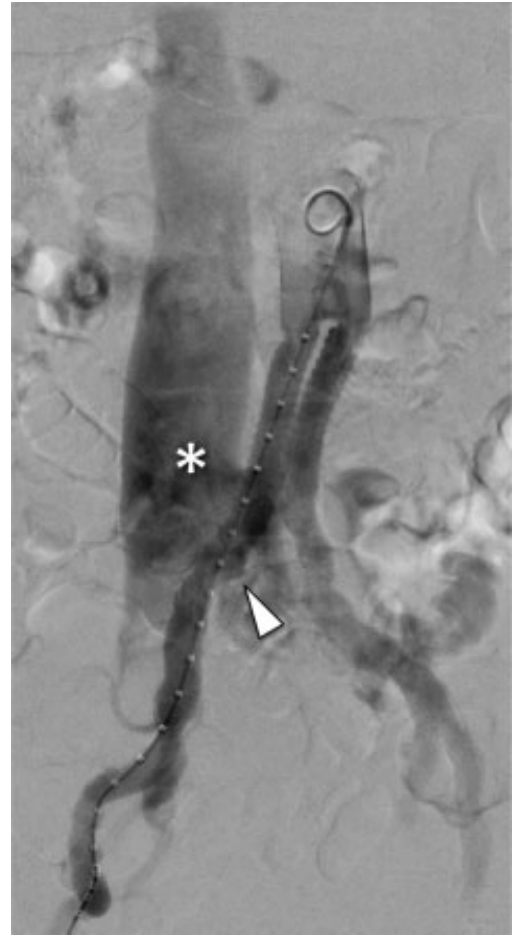

Fig. 2 Abdominal digital subtraction angiography that confirms the fissured pseudoaneurysm (arrowhead), leading to right iliac arteryinferior vena cava $\left({ }^{*}\right)$ arteriovenous fistula. anastomotic pseudoaneurysm must be treated to prevent its possible rupture and cardiac decompensation due to left-toright shunting. Treatment options include endovascular techniques or open surgical management. Nowadays, endovascular techniques are the preferred modality for most clinicians. ${ }^{3}$

\section{Conflicts of Interest}

The authors declare no conflict of interest related to this article.

Funding

This case was funded solely through institutional sources.

\section{Acknowledgements}

None.

\section{References}

1 Fukushima RB, Wolosker N, Benitti DA, Puech-Leão P. Endovascular treatment for iliac artery pseudoaneurysm with arteriovenous fistula after abdominal aortic aneurysm open repair. Clinics (Sao Paulo) 2011;66(08):1499-1500

2 Fuentes-Orrego JM, Pinho D, Kulkarni NM, Agrawal M, Ghoshhajra BB, Sahani DV. New and evolving concepts in CT for abdominal vascular imaging. Radiographics 2014;34(05):1363-1384

3 Nakad G, AbiChedid G, Osman R. Endovascular treatment of major abdominal arteriovenous fistulas: a systematic review. Vasc Endovascular Surg 2014;48(5-6):388-395 